\title{
AGENT-BASED SIMULATIONS TO DETERMINE MEDITERRANEAN FRUIT FLY DECLARATION OF ERADICATION FOLLOWING OUTBREAKS: CONCEPTS AND PRACTICAL EXAMPLES
}

\author{
N. C. MANOUKIS AND T. C. COLLIER \\ Tropical Crop and Commodity Protection Research Unit, Daniel K. Inouye United \\ States Pacific Basin Agricultural Research Center, United States Department of \\ Agriculture - Agricultural Research Service, Hilo, Hawaii 96720 USA; \\ Nicholas.Manoukis@USDA.GOV
}

\begin{abstract}
SUMMARY
Areas of the world that do not have established populations of the Mediterranean fruit fly Ceratitis capitata (Wiedemann) and other invasive pestivorous Tephritidae are sometimes subject to incursions due to increasing travel and trade. When these occur, control programmes are put in place often including quarantine and additional measures until eradication of the outbreak is declared. A critical practical question that arises is how long to maintain the eradication programme and associated area-wide measures after the last sampling of the invading Mediterranean fruit fly. Current practice is usually to maintain measures and increased monitoring until enough time has passed for three generations of flies without another fly catch; generation times are calculated via thermal unit accumulation ("Degree Day"). A recent alternative or complementary approach is to model the invading population using an Agent-Based Simulation (ABS). This chapter outlines the use of MEDiterranean fruit Fly Outbreak and Eradication Simulation (MEDFOES), an ABS implementation aimed at modelling invading Mediterranean fruit fly populations to determine effective duration of quarantine and other eradication measures following the last detection of an incursion. Basic concepts are described, together with a description of major functions and use of thousands of individual simulations to encompass the range of demographic possibilities. Finally, specific examples from Santiago, Chile and California, USA are offered to show how the ABS can provide useful information for programme managers setting eradication programme durations.
\end{abstract}

Key Words: Invasion biology, incursions, outbreaks, quarantine, eradication, Tephritidae, Ceratitis capitata, medfly, monitoring, detections, California, Chile, degree-days, model, modelling, individualbased simulations, multi-agent simulations, MED-FOES Development and Field Application, pp. 869-888. CRC Press, Boca Raton, Florida, USA. (C) 2021 U. S. Government 


\section{INTRODUCTION}

Incursions of the polyphagous pest Mediterranean fruit fly Ceratitis capitata (Wiedemann) occur in urban and agricultural areas around the world. The Global Eradication and Response Database (GERDA), although not a complete compilation of all invasions and eradication efforts, currently lists 117 Mediterranean fruit fly incursions and eradication programmes in a wide variety of geographic areas, from the USA to Australia, Chile, Mexico, and New Zealand among others. Responding to each of these outbreaks costs an average of USD 12 million (normalized to 2012 USD) (Kean et al. 2012; Suckling et al. 2016). While this cost is small compared with the estimated damage from a Mediterranean fruit fly establishment in many of these areas (USD 1500 million in the US state of California alone [Siebert and Cooper 1995]), it is still important to optimize responses to incursions. This is because mounting and maintaining an effective response and quarantine is a significant burden and organizational challenge for state and private organizations.

Quarantine measures are often put into place following the detection of an incursion of an invasive pest (FAO 2016a). In the case of Mediterranean fruit fly, quarantine measures involve designating an area around the find where fresh fruits are restricted from leaving. If the area includes commercial host fruit production, then the quarantine measures can result in serious economic losses. Therefore, the period to maintain the quarantine and associated measures against the invading Mediterranean fruit fly population becomes a critical practical question.

Current practice in many parts of the world is based on ISPM 26 (FAO 2016b), such as the protocol used by the California Department of Food and Agriculture (CDFA), whereby the quarantine is suspended when three full generations of the fly have passed without another find; generation times are calculated via thermal unit accumulation (California Code of Regulations 2017). The thermal accumulation development models most often used simply posit that development from egg to adult requires accumulation of a specific amount of heat above a base threshold, measured in units of degree-days (Roltsch et al. 1999). Various calculation methods exist, but all approximate the integral of temperature over time for temperatures above a given base temperature. The simplicity of degree-days calculations is attractive, and thermal accumulation models are widely used with impressive accuracy for predicting developmental timing in many agricultural contexts. However, it may not be entirely appropriate for Mediterranean fruit fly eradication and quarantine duration determination. "Degree-days" is a development model, not a population or eradication model. Moreover, the requirement for three generations of degree-days to pass is difficult to justify theoretically.

An alternative approach to estimating eradication programme length or duration since the last detection is to use an Agent-Based Simulation (ABS; also called "Individual-Based" or "Multi-Agent") to simulate the arrival of C. capitata in a new area as an insect invasion (Manoukis and Hoffman 2014). In an ABS, individual flies are described as unique and autonomous, and where they usually interact with each other and their environment at a local level (Railsback and Grimm 2012). An important characteristic is that individual members of the simulation ("agents") are represented independently in computer memory via the simulation software, and these 
have behaviours (functions) and characteristics (parameters) that may make them unique. The behaviours and characteristics of the entire system are not explicitly coded by the programme rather they emerge from the interactions and behaviours of the system's constituent agents.

The ABS approach has not received a lot of attention for studies on insect invasions (Crespo-Pérez et al. 2011; although see Vinatier et al. 2011 for an example of an ABS of an agricultural pest), but it has been more widely used to address questions on physiological ecology, foraging networks, ant nest choice, and disease vector dynamics among others (Jackson et al. 2004; Pratt et al. 2005; Almeida et al. 2010; Radchuk et al. 2013).

This chapter is concerned with the concepts, implementation and use of an ABS entitled MED-FOES (MED-Fly Outbreak and Eradication Simulation; MED-FOES 2019), that was designed to simulate the invasion, programmatic response to an outbreak, and extirpation of a population of Mediterranean fruit fly in a pest free area (Barclay et al. 2021). The original goal of the ABS and its implementation is to provide an estimate of eradication programme duration that is roughly independent of the values determined by thermal unit accumulation.

In an area-wide context, which means addressing a total pest population within a defined area and not a localised field-by-field approach (Hendrichs et al. 2007), modelling eradication programme lengths is important for several reasons:

First, programmes against Tephritidae tend to encompass large areas, and many of the measures taken following a Mediterranean fruit fly incursion are likewise applied over these same vast areas, such as intense surveillance, restriction of fruit movement and in most cases SIT application. These approaches are not effective in an uncoordinated property-by-property setting.

Second, there are area-wide programme costs to be considered; these can grow to be large if excessively long programmes are implemented, in particular if quarantines and commercial production areas are involved.

Third, eradication efforts are often directly connected to area-wide programmes (Smith 1998; Myers et al. 2000). If eradication programme lengths against Mediterranean fruit fly are too short, there is potential for survival of remnants of the invading population, which would require a new eradication campaign or in a worst case scenario, result in the establishment of the pest species in a free area (Carey 1991; Papadopoulos et al. 2013; McInnis et al. 2017; Shelly et al. 2017).

MED-FOES is based on a modelling framework that simulates the process of Mediterranean fruit fly population extirpation/eradication (Manoukis and Hoffman 2014). A description of the important functions included in the simulation is given in Section 2, with minimal mathematical background. The use of thousands of simulations to understand the range of possible outcomes following an outbreak is the focus of Section 3. In Section 4, two examples of historical incursions are analysed to illustrate the use of MED-FOES for determining eradication programme length. Finally, Section 5, includes broad conclusions and some suggestions for future work. The actual installation and mechanics of using the software are not covered here; the reader is referred to the software manual for these details (MED-FOES 2019). 


\section{CONCEPTS AND IMPLEMENTATION}

\subsection{Simulation Description}

Mediterranean fruit fly agents in the simulation can be created, and they develop, reproduce and die. The major challenge is to have the agents behave in a biologically realistic manner so that the results of thousands of executions of the simulation can be usefully related to flies in the real world.

Figure 1 gives a graphical representation of the developmental stages and states fly agents in MED-FOES can occupy as well as their connections. During each hour of the simulation, flies may move from one developmental stage to another, or not. They also may go from being alive to being non-reproductive (mated with a sterile male) or dead, when they are removed from the simulation and no longer considered.

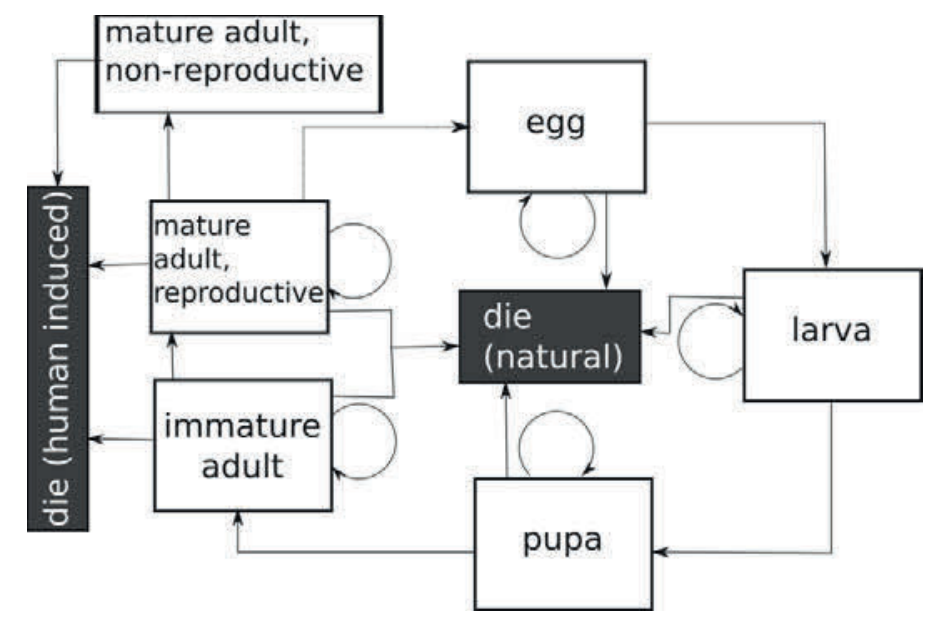

Figure 1. Graphical representation of the developmental stages and states of Mediterranean fruit fly agents in MED-FOES. Arrows indicate changes in stage or state per time step (adapted from Manoukis and Hoffman 2014).

\subsection{Initialization}

The simulation is initialized based on an estimated number of potential remnant adult female flies in the area of an incursion that has been subject to area-wide application of eradication measures. Given this number, the estimated number of female flies in all other stages is determined based on the expected stable age distribution for the Mediterranean fruit fly from the literature (Carey 1982; Vargas et al. 1997). These flies are then iterated through hourly time steps; for each time step, they may develop, reproduce, die of natural causes or die of human-induced causes.

The current version of MED-FOES includes a simple model of the initial stages of the simulation. There is a time between when a detection that exceed the threshold needed to trigger a response during which full counter-measures are not in place. In general, the number of flies detected until the point of declaration is used to estimate 
the initial population size range. During the short period between detection exceeding threshold and counter-measure implementation (2-5 days in many jurisdictions), the population of agents can grow, and no human-induced mortality is considered. At the time $R$ (in days) counter-measures initiate and the population begins its demographic trajectory to eradication. This representation of the early stages is unrealistic, as in real incursions detections may occur asynchronously, trapping densities may be changed in response, and individual counter-measures are applied at different times. However, the simulations are generally not highly sensitive to the length of $R$ or initial population size (Manoukis and Hoffman 2014). Future versions of MED-FOES are planned to have the ability to include more realistic initial stages and the ability to model time varying interventions.

\subsection{Hourly Temperatures}

The only time-dependent input to the ABS is an hourly air temperature time series. Ideally these data should be acquired from a long-term weather station in close proximity to the outbreak being simulated. Data from airports archived in the Integrated Surface Data repository of the National Oceanic and Atmospheric Administration (NOAA) are often appropriate and methods for processing them have been published (Collier and Manoukis 2017).

Care should be taken to ensure that the temperature data are accurate as the ABS (as well as simpler thermal accumulation models) can be sensitive to biases and errors in the data. For example, a sensor improperly shielded from the sun would tend to report slightly higher temperatures, and the cumulative nature of a thermal accumulation development model would amplify the effect over time. Similarly, spurious very high or very low values produced by sensor errors could lead to erroneous high mortality events in the ABS. Actual temperature data often contain gaps, errors, and a non-uniform sampling rate, and therefore require some 'cleaning' before they can be used.

A cleaning process typically starts with the detection and removal of erroneous outlier values. A large variety of methods exist for outlier removal, but a simple method which works well for temperature time series data is computing the running standard deviation $(\sigma)$ over a window spanning several days, computing the running median $(m)$ over a several hour window, and rejecting individual temperature values $(t)$ for which the absolute value of $(t-m) / \sigma$ exceeds a predefined threshold.

The next step of cleaning is the identification and filling of gaps in the data. Because of the daily cycle of temperature data, it is appropriate to use different methods to fill small (less than a few hours) and large gaps (a few hours to several days). Small gaps may be filled by simple interpolation, which can also serve to simultaneously resample the data to a true hourly frequency. Large gaps, however, are more appropriately filled by interpolating across observations from the same time of day across days. Gaps larger than several days would likely require special treatment dependent on the particulars of the location and season. If actual hourly temperature data are not available, then an approximation may be inferred from daily minimum and maximum temperatures using established methods such as the one implemented in the "TemperatureEstimator.java programme" distributed with MEDFOES (Reicosky et al. 1989; Campbell and Norman 1997). 


\subsection{Development}

The single factor that determines the probability of stage transition or ovarian maturation (development) for flies in the simulation is temperature. The simulation requires a base developmental temperature $T_{\min }$ and time-to-transition $K$ for each of the following transitions: egg to larva, larva to pupa, pupa to adult and adult to mature adult.

Several reports in the literature give the mean time to transition $(d)$ for these stages as affected by a range of constant fixed temperatures $(T)$. The common practice is to regress the developmental rate $(=1 / d)$ against fixed temperatures, which gives a clear linear relationship for temperatures between about 16 and $30^{\circ} \mathrm{C}$. The linear regression model would then be

$$
\frac{1}{d}=a+b T
$$

where $a$ is the intercept and $b$ the slope. The parameters required by the simulation are then calculated as follows:

and

$$
T_{\min }=-\frac{a}{b}
$$

$$
K=\frac{1}{b}
$$

There are two methods available for calculating the probability of stage transition. The simplest is a "uniform" model, where the probability of transition at any given hour is determined only by the temperature during that hour. For each hour, a random number between zero and one is drawn from a uniform distribution for each insect that is not a mature adult. If this number is lower than the developmental rate for that temperature, then a stage transition occurs. The average time for a transition at a given temperature is thus equal to $1 / d$.

Note that the "uniform" method described above does not take into account the time each insect has spent in a given stage. Therefore, it is possible (though unlikely for most parameter combinations and realistic temperatures) that an agent could go from being an egg to an adult in a few hours. A more realistic developmental model is the "thermal summation" approach (Fletcher 1989), where each degree above $T_{\min }$ for each hour counts towards a required threshold $\mathrm{C}$ for stage transition. Variation is included for each individual fly agent when it is created in the form of a variable $\gamma$, which is the standard deviation of the variation in development time as a proportion of the development time for each stage. Thus, when

$$
C+\gamma<\sum_{t=0}^{i} T_{i}-T_{\text {min }}
$$

from the time of insect creation ( 0 ) to the current time $i$, stage transition occurs. Note that the value of $C$ is stage-specific, and $\gamma$ constant across stages. 


\subsection{Mortality}

There are two ways that death is implemented in the simulation. The simplest approach is to set a fixed stage-specific daily death rate, denoted as $M_{x}$. Each hour of the simulation, for each insect at stage $x$, a random double precision floating point number between 0 and 1 is drawn and if it is lower than $M_{x}^{1 / 24}$, then the insect dies. This method is completely temperature-independent and is a useful approximation when the effect of temperature on mortality is unknown.

In the case of the Mediterranean fruit fly reliable data are available on the effect of temperature on daily mortality rates. MED-FOES uses the stage-specific quadratic relationships from Gutierrez and Ponti (2011) for this relationship. When this mode it used, the run-specific parameters $M_{x}$ are the death rate at the optimum temperature $\left(20-25^{\circ} \mathrm{C}\right)$ rather than the average mortality per unit time.

Additional mortality on adults is introduced at a given number of days after the simulation starts. The probability of human-induced death is fixed per simulation and the same for mature and immature adults; it represents human-induced mortality as a result of counter measures. Human induced mortality is currently limited to the adult stage, though some control measures, such as fruit stripping, may affect immature flies. After human intervention, it is optionally possible to simulate the effect of trapping separate from mortality induced by other countermeasures. This is discussed in the next Section.

\subsection{Trapping}

Spatially explicit consideration of the effect of trapping on agent mortality is possible as of MED-FOES version 0.6, using the approach of Manoukis et al. (2014) as implemented in the software "TrapGrid" (Manoukis et al. 2014). For parameters relevant to the Mediterranean fruit fly, see Manoukis et al. (2015). A brief description of the trapping model is given here.

TrapGrid is an implementation of a landscape-level, spatially explicit model of trap networks that incorporates variable attractiveness of traps and a movement model for dispersion. TrapGrid simulates susceptible insect capture by placing traps in a rectangular area. Each trap has a parameter indicating its attractiveness $(\lambda)$. Using this value, the escape probability for a given insect at a given distance from the trap can be calculated. The calculation of escape probability can be conducted for many points in the trapping grid, yielding an instantaneous estimate of the escape probability. Note that probability of capture is simply 1 - \{probability of escape .

We calculate the distance to a given trap as:

$$
d=\sqrt{\left(x_{t}-x\right)^{2}+\left(y_{t}-y\right)^{2}},
$$

where $\left(x_{t}, y_{t}\right)$ is the position of the trap and $(x, y)$ is the position of the fly. For $d$ $>=0$, we use an exponential decay with a logistic $(H(d))$ to model the probability of being captured:

where

$$
p=f(d, \lambda)=\lambda e^{-\lambda d} H(d)
$$

$$
H(d)=\frac{1}{1+e^{-2 \lambda d}}
$$


These can be combined, producing the hyperbolic secant:

$$
f(d, \lambda)=\frac{2 e^{\lambda d}}{1+e^{2 \lambda d}}
$$

Fig. 2 shows how the probability of capture changes with distance from the trap given $\lambda$. Each trap in a TrapGrid model represents the spatial relationship between distance from a lure-baited trap and probability of capture in the very near future. The parameter $\lambda$ is the attractiveness of the trap, with smaller values representing a more attractive trap.

One important feature of the capture model used is that, for a given value of $\lambda, 1 / \lambda$ is the distance at which there is a $65 \%$ chance of capturing a susceptible insect. This allows easy comparison of trap attraction between species and lures. Movement in TrapGrid as used by MED-FOES is simple diffusion (Skellam 1951; Kareiva 1983), through which the probability of capture over time $\left(p_{t}\right)$ is calculated.

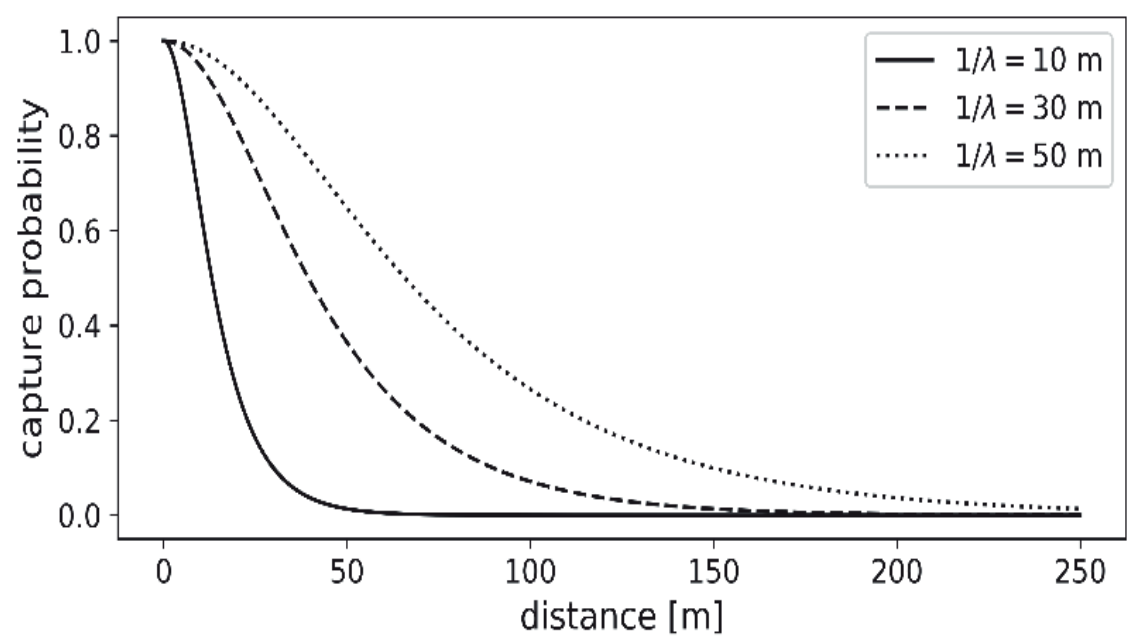

Figure 2. Capture probability for an individual insect versus distance from a trap, where attraction $(1 / \lambda)=10$ (solid), 30 (dashed) and 50 (dotted) meters. $1 / \lambda$ represents the distance at which there is a 0.65 probability of capture (adapted from Manoukis et al. 2014).

In order to make an estimate of $p_{t}$, consider the net movement of a fly over the time period in question (usually a day), represented by the parameter $D$ in diffusion models. Estimates of $D$ from the literature are around $1 \times 10^{4} \mathrm{~m}^{2} /$ day (Corbett and Plant 1993). We can use the net movement per day to model the proportion of the arena space that an individual Mediterranean fruit fly might "experience" per day.

The diffusion in two dimensions is well studied, and has the following form:

$$
\frac{d N(x, y, t)}{d t}=D\left[\frac{d^{2} N}{d x^{2}}+\frac{d^{2} N}{d y^{2}}\right]
$$


where $N$ is the population density, $t$ is time, $x, y$ are spatial coordinates and $D$ is the diffusion coefficient. This process produces a bivariate normal distribution of density over time, the probability density function (pdf) of which is the basis of our model of the fly population and its spread over time:

$$
g(x, y)=\frac{1}{2 \pi \sigma^{2}} e^{-\frac{1}{2}\left(\frac{x^{2}}{\sigma^{2}}+\frac{x y^{2}}{\sigma^{2}}\right)}
$$

Here, $\sigma$ is the standard deviation, assuming that

$$
\mu=0, \sigma x=\sigma y=\sigma \text { and that } \operatorname{cor}(x, y)=0 .
$$

Further description is beyond the scope of this document, but it is important to note that the diffusion parameter $D$ in $\mathrm{m}^{2}$ per day is the single factor that determines how quickly the simulated flies in TrapGrid will spread.

MED-FOES can run a set of TrapGrid simulations before creating agents. This set of spatial simulations is used to determine the average daily probability of death from trapping given a trapping network following a detection, and then this mortality is scaled to hourly time steps and applied.

\subsection{Reproduction}

Every mature reproductive Mediterranean fruit fly will oviposit eggs every 24 hours prior to intervention time $t_{s}$. In this sense, the simulation only includes females. The mean number of eggs and variance in reproductive output are set by the variables $r$ and $r_{\text {var }}$. After human intervention, reproduction may be curtailed at a set daily rate, which is denoted $r_{\text {red }}$. This variable is included based on modelling of the Sterile Insect Technique (SIT) and its effects on host populations (Knipling 1979).

In the $\mathrm{ABS}$, each mature adult fly that exists at $t_{S}$ is subject to loss of reproductive ability each day with probability $r_{\text {red }}$. In the case studies below, flies emerging after $t_{S}$ are assumed to not be able to reproduce. Mating is currently not required for reproduction, so the difficulty in finding a mate that comes with small population sizes is not modelled. In addition, the Allee effect, where there is reduced mean individual fitness in small populations, is also not considered.

\section{ANALYSING AN OUTBREAK: MULTIPLE SIMULATIONS}

Until this point, we have focused on a single simulation and how biological and population processes are represented. Individual simulations can vary from one to the next, even if the same parameters are used, due to stochasticity (random events) built into the model. However, in order to obtain useful and actionable information on a real-world incursion, a single set of parameters is insufficient because there is uncertainty on the values of critical parameters. Thus, it is necessary to execute a set of simulations, varying parameters between runs, in order to obtain the range of possible outcomes following an incursion. MED-FOES has this functionality built-in via a separate executable programme called "med-foes-p.jar" (MED-FOES 2019). 


\subsection{Exploring the Parameter Space}

MED-FOES includes a large number of parameters that may be set over a range, including aspects of mortality, development, reproduction, and control measures. Even if a fixed number of discrete levels over all these ranges were selected, a full factorial experiment would be computationally prohibitive.

Latin Hypercube Sampling (LHS) is used to select parameter sets through the parameter hyperspace (Blower and Dowlatabadi 1994). The details of LHS are beyond the scope of this document, save that it ensures an even distribution of the combinations of parameters used for individual runs.

The number of simulations executed can be set by the user. A number in the thousands will usually provide sufficient resolution on the range of possible outcomes.

\subsection{Summarizing Output}

MED-FOES produces a summary output file that is named MEDFOESp_\{timestamp\}_summary.txt, where $\{$ timestamp $\}$ is the date and time the programme was run to avoid inadvertently overwriting previous output. This file contains the parameters used as well as results, including the mean, standard deviation, and 25, 50, 95, and $99 \%$ quantiles of the length of runs (individual simulations). Since runs nominally start close to the time of last detection and terminate upon eradication of the simulated population, these run length statistics summarize time to eradication across the various parameter sets tested.

Additionally, the mean and standard deviation of number of flies at the end of the simulations are reported and can be used to detect situations where some runs ended without reaching eradication due to an insufficient amount of input temperature data or exceeding the maximum number of flies allowed. Summary figures are produced that give a quick visual summary of the outcomes of the set of simulations (Fig. 3).

A MED-FOESp_\{timestamp\}_details.txt file is also produced. This is a tabdelimitated table reporting for each run the length of the run in simulated hours, the end condition (eradication, out of temperature data, or maximum flies exceeded), and total number of flies at the end. From this file, arbitrary statistics for the time to eradication can be produced.

Finally, for each individual run a summary and details file is produced under the "runs" directory. The summary file gives the specific parameters used for that particular simulation, in contrast to the MED-FOESp summary file, which gives the ranges sampled by the LHS procedure. It also gives summary results: number of hours simulated, number of flies at the end of the simulation, cumulative number of eggs, and cumulative number of deaths.

The details file is a comma-delimited file with a row for each day of the simulation containing: time, mean temperature, minimum temperature, maximum temperature, cumulative death, cumulative birth, the number of flies in each life stage (egg, larvae, pupae, adult), and total number of flies. The collection of runs details files can be used to produce a wide variety of detailed outputs, such as Figs. 4 and 5 shown in the next Section. 

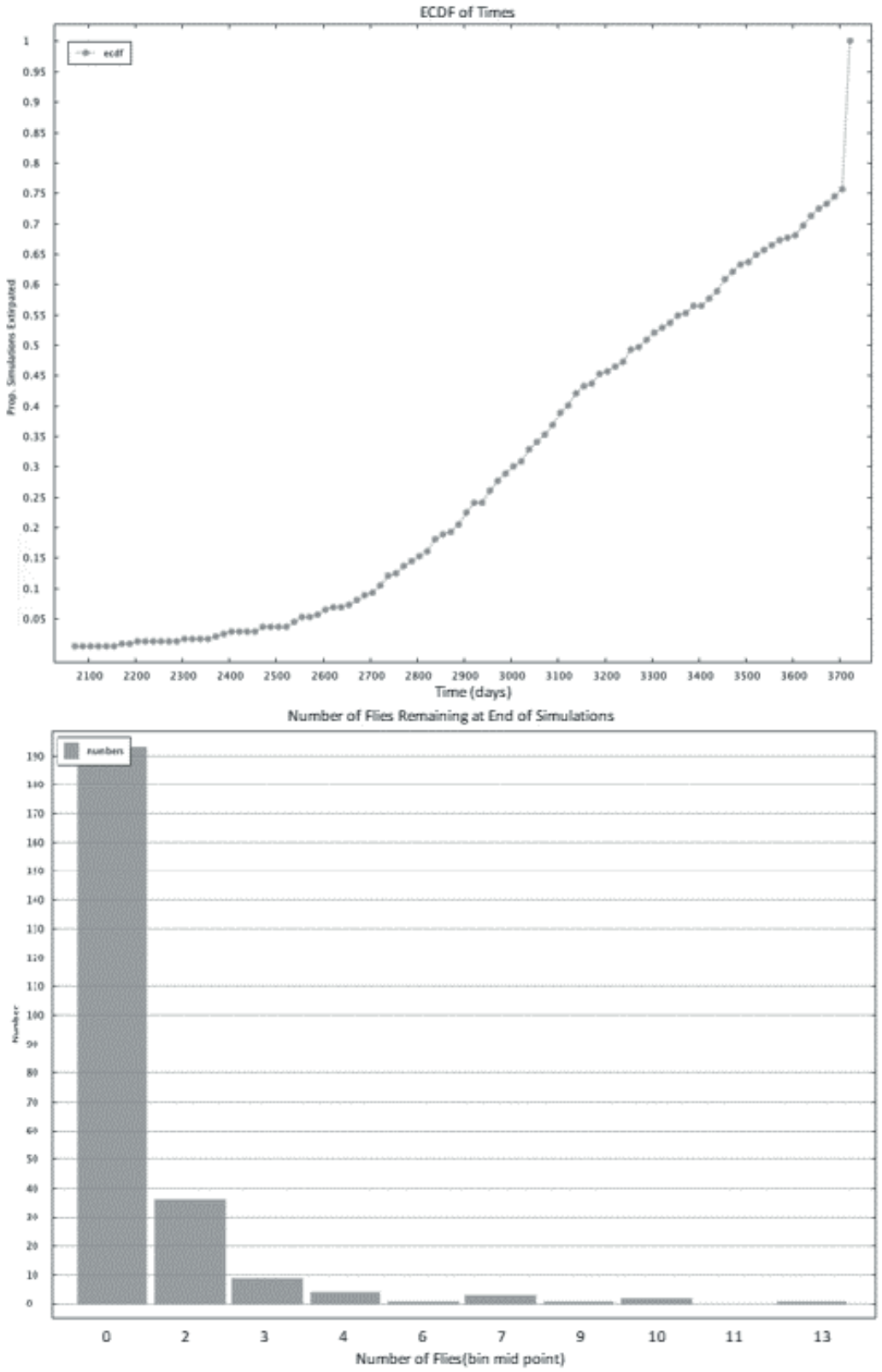

Figure 3. Example of graphical output from MED-FOES showing 250 runs simulating an outbreak of Mediterranean fruit fly in California. Upper panel: Empirical cumulative frequency distribution of the number of simulations showing extirpation over time. Bottom panel: Histogram of the number of surviving agents at the end of the 250 simulations. This simple summary graphic can be used in internal reports without the need of post-simulation data manipulation. 


\section{PRACTICAL EXAMPLES}

\subsection{Santiago, Chile}

Chile is internationally recognized to be free of tephritids of economic importance, including species in economically important genera Anastrepha, Bactrocera, and Ceratitis. This is facilitated by Chile's geographic isolation, stemming from the presence of the Pacific Ocean to the West and the Andes mountains in the East, as well as its excellent pest exclusion infrastructure. Despite this isolation, occasionally Mediterranean fruit fly outbreaks are detected in the country, usually via the network of about 14000 traps.

One such detection occurred in the neighbourhood of Independencia in Santiago, in 2011. The first fly was detected in a Jackson trap baited with trimedlure on 14 October, and the response including quarantine, enhanced trapping, bait spray (GF120) applications, soil treatment under host trees, and fruit stripping was put in place by 18 October. Another fly was sampled on 18 October in a McPhail trap about 800 $\mathrm{m}$ from the first detection; thereafter, no further detections of adults were made. The response programme was concluded on 9 February 2012 after three generations $\left(\mathrm{F}_{3}\right)$ of degree-day development as calculated by the Chilean Agricultural and Livestock Service (SAG) using the method of Tassan et al. (1983). This occurred 115 days after the first find.

For the ABS analysis, conducted with version 0.6.2 of MED-FOES, critical parameters that had to be evaluated included: initial population size, reduction in fecundity over time, and hourly temperature data. Starting with the third of these, hourly temperature data were acquired from NOAA's online ISD-Lite dataset derived from the Integrated Surface Database for the weather station at the nearby (approx. $12 \mathrm{~km}$ from the outbreak neighbourhood) Comodoro Arturo Merino Benítez International Airport (SCEL). The air temperature data for one year starting on 18 10-2011 (day 0) were extracted and cleaned using the method described previously and saved to a comma separated format (csv) file for MED-FOES to use as input.

The SAG programme did not include SIT for the 2011 outbreak. This might mean that the modeller decides not to include the parameter on fertility reduction per day ("rred"). However, due to the intense fruit stripping (100\% in a $400 \mathrm{~m}$ radius from each find) plus the soil drenches (not used in California currently), it was estimated that there was probably an effective reduction in the probability of reproduction over a wide range, 0.2-1.0.

Finally, the initial population size was estimated based on the surveillance network in Chile at the time of the outbreak. This consisted of one trimedlure trap per 25 ha and one protein trap per 100 ha, comparable to the California values of one trimedlure and one protein trap per 52 ha. This gives a rough detection sensitivity of 2-3\% of the adult population (Manoukis and Hoffman 2014). Assuming a stable age distribution (from Table 3 in Carey 1982), gives estimated numbers in the other life stages. Though these figures are rough, they are probably sufficient since the model is not very sensitive to initial numbers (Manoukis and Hoffman 2014). 
The complete set of parameters used to generate the runs are given below:

\begin{tabular}{|c|c|c|}
\hline $\mathrm{T}$ & SCEL_2011-10-18.csv & Hourly temperature data file \\
\hline $\mathrm{Ni}$ & 66,100 & Initial population size (range) \\
\hline Ad & $29.8,49.7,15.6,1.8,3.1$ & Initial age distribution (from Carey 1982) \\
\hline $\mathrm{R}$ & 4 & Days before intervention is implemented \\
\hline S & $0.05,0.15$ & Daily human-induced mortality (range) \\
\hline rred & $.2,1$ & Reduction of reproduction (range) \\
\hline Sai & true & Sterility after intervention \\
\hline TEL & $9.6,12 \cdot 5,27.27,33.80$ & Transition parameters, egg to larva (range) \\
\hline TLP & $5.0,10.8,94.50,186.78$ & Transition parameters, larva to pupa (range) \\
\hline TPA & $9.1,13.8,123.96,169.49$ & Transition parameters, pupae to adult (range) \\
\hline TIM & $7.9,9.9,58.20,105.71$ & $\begin{array}{l}\text { Transition parameters, adult to sexually mature } \\
\text { (range) }\end{array}$ \\
\hline Me & $0.0198,0.1211$ & Daily natural mortality of eggs (range) \\
\hline Ml & $0.0068,0.0946$ & Daily natural mortality of larvae (range) \\
\hline $\mathrm{Mp}$ & $0.0016,0.0465$ & Daily natural mortality of pupae (range) \\
\hline $\mathrm{Ma}$ & $0.0245,0.1340$ & Daily natural mortality of adults (range) \\
\hline $\operatorname{tdm}$ & true & Use temperature dependent mortality \\
\hline $\mathrm{r}$ & 5,35 & Eggs produced per reproduction event (range) \\
\hline rvar & 3.57 & Variance in eggs produced per event \\
\hline $\mathrm{Dm}$ & 1 & $\begin{array}{l}\text { Development model; } 0=\text { uniform, } 1=\text { thermal } \\
\text { summation }\end{array}$ \\
\hline TuSD & 0.05 & Variation in thermal unit transition \\
\hline Tmax & 35 & Maximum temperature for development \\
\hline o & Run_2011-10-18 & Output directory for results \\
\hline $\mathrm{nR}$ & 2500 & Total number of simulations to run \\
\hline nT & 20 & Number of threads to employ \\
\hline Mx & 500000 & Maximum number of flies allowed \\
\hline seed & 4354885 & Random number seed \\
\hline q & true & Suppress progress output to terminal \\
\hline pr & false & Produce only LHS parameters \\
\hline plot & false & Generate summary plots \\
\hline
\end{tabular}

The parameters above when executed on a command line on a computer with a quad-core processor would be invoked roughly as follows (for more details please refer to the programme manual, distributed with MED-FOES):

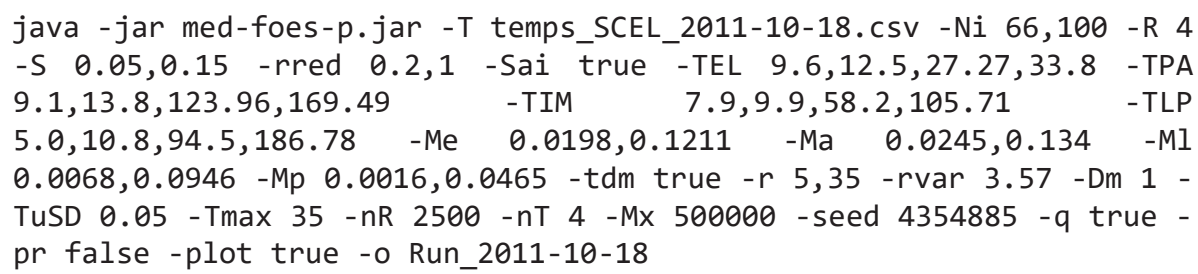


The parameters and ranges above were used to execute 2500 runs. The number of living individual agents per day and mean trend from 100 of these simulations (reduced from 2500 for clarity) is shown in Fig. 4.

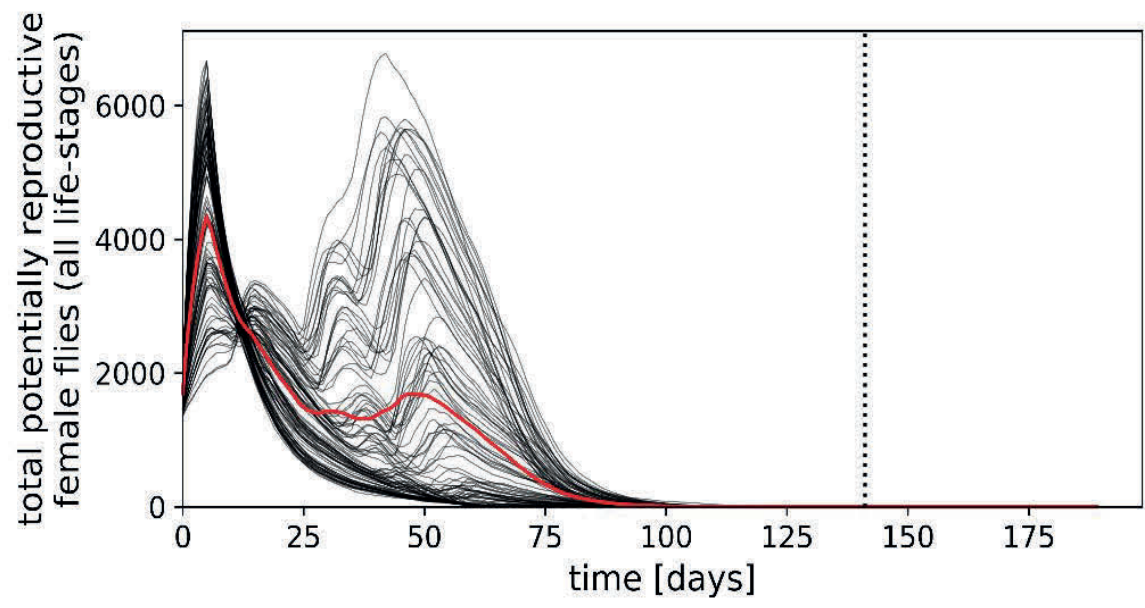

Figure 4. Total number of potentially reproductive female flies including all life stages for 100 simulations, last fly was detected 18-10-2011 (day 0), Santiago, Chile. Mean trend is shown by the red line. The dashed vertical line shows the point at which 95\% of the simulations show eradication (141 days).

Fig. 4 shows some interesting characteristics, including parameter sets that seem to lead to increasing population sizes through a second and even third generation. This can be interpreted as a function of favourable temperatures for initial adult survival and reproduction, and for immatures to progress to adulthood from the initial population and from eggs laid before time $R$. Clearly a maximum population size of nearly 7000 individuals is a maximal (and unlikely) scenario, but it is indicated by a small number of the parameter sets simulated; the average time from declaration to eradication was 109 days.

In terms of indicated duration of the quarantine and other control measures, $95 \%$ of the simulations showed eradication after 141 days, $18 \%$ longer than the $\mathrm{F}_{3}$ degreeday calculation that led to the actual 115-day control and quarantine period.

It is common for the ABS to predict longer durations than simple thermal accumulation models for outbreaks persisting through the summer in temperate areas. Since the ABS incorporates other factors which may mitigate the rapid generational turnover shown by thermal accumulation in hot weather, or the near halt in development seen in cold weather such as illustrated by the next example, it will often show less extreme seasonal swings than simple degree-day models (Collier and Manoukis 2017). 


\subsection{Perris, California USA}

California has been the site of multiple $C$. capitata detections and outbreaks over the last four decades or so, leading some to propose that the species and other Tephritidae are established there (Carey 1991; Papadopoulos et al. 2013). However, this theory has not been accepted by most phytosanitary experts, internal and external California trading partners, or customers of horticultural commodities (McInnis et al. 2017; Shelly et al. 2017). As in Chile, California maintains a large (>90 000) trapping network for detecting Mediterranean fruit fly incursions. Additionally, since 1996 California has been conducting a preventive sterile male release programme over the high-risk Los Angeles Basin, where most of incursions of this pest are detected.

In December of 2014, Mediterranean fruit fly was detected in the city of Perris, that is located in Riverside County east of Los Angeles. The city is about $27 \mathrm{~km}$ outside the zone the preventive release programme covers. The initial detection was a find of two unmated adult females on 10 December 2014 in McPhail traps, which was followed by eight other finds in the same residential area over the next few weeks, including a find of larvae on 14 December 2014. The final find occurred on 29 December 2014. Mitochondrial genotyping indicated the AAAB mitotype consistent with a Central American source.

Eradication efforts started quickly, within one day, and included fruit removal, spinosad foliar bait spraying, and inundative releases of approximately 1.5 million sterile male flies every three or four days. The total quarantine area established was $215 \mathrm{~km}^{2}$, with the sterile male releases targeting a $33.4 \mathrm{~km}^{2}$ core area.

The weather station at March Air Reserve Bases (KRIV) is approximately $12 \mathrm{~km}$ from the find sites. This station has good data going back to the 1940s, available through NOAA's ISD archive, which allows not only modelling the 2014 outbreak, but also putting it in the context of how a similar outbreak would have progressed if it started on the same day of the year in previous years. Specifically, data from the ISD-lite data from 1950 through 2015 were cleaned as described earlier in this chapter and used to run MED-FOES simulations.

MED-FOES v0.6.2 was run with the same parameters as the Santiago model, except for the initial population (Ni) of 25 to 133 adult females, a delay before the start of SIT releases $(\mathrm{R})$ of 1 day, and the input temperature data. By day 151 after the last fly was detected, $95 \%$ of the ABS simulations predicted eradication (Fig. 5). This is more than one month shorter than the quarantine and control period that was actually implemented of 189 days, or 187 days produced by recalculating the degreeday based three generation time $\left(\mathrm{F}_{3}\right)$ using the same KRIV temperature data used for the ABS.

In contrast to the ABS results for the 2011 Santiago outbreak, the 2014 Perris, simulations show no evidence of large population sizes after control measures were started. There is a very small increase in number of flies around day 80 for a few of the simulation parameter sets, but the overall character is rapid decline followed by a small population resisting final eradication for a relatively long time. Remembering that any eggs laid after time $R$ (counter-measure start) are sterile, only agents that existed before that time could reproduce and account for population growth later in the simulations. Unfavourable environmental conditions for those individuals, leading 
to their rapid and early mortality, would cause the difference observed in overall numbers between this case and the Santiago case presented above.

The ABS results indicate eradication occurring significantly earlier than the simple thermal accumulation calculation (151 vs 187 days). This is a common finding for outbreaks covering cold periods in temperate climates, since cold temperatures in the degree-day model just slow development. In the ABS model, however, mortality occurs even with slow development and may even be increased due to particularly cold periods.

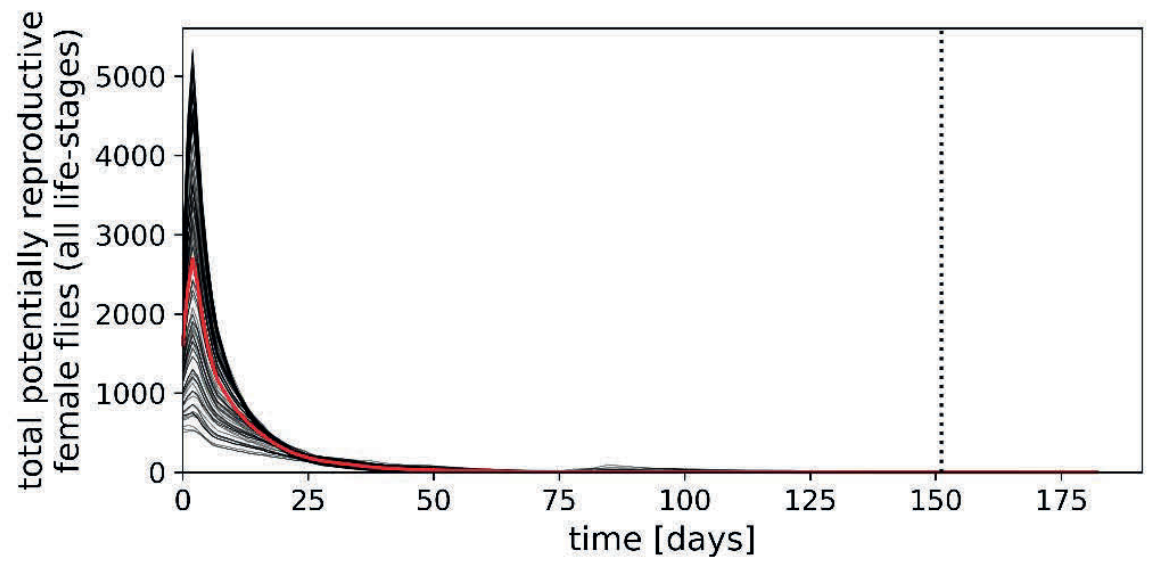

Figure 5. Total number of potentially reproductive female flies including all life stages for 100 simulations, last fly was detected 29-12-2014 (day 0), Perris, California. Mean trend is shown by the red line. The dashed vertical line shows the point at which $95 \%$ of the simulations show eradication (151 days).

In addition to simulating the particular outbreak, the ABS can be used to simulate what would happen if the same outbreak occurred at a different time. One application of this is to simulate the outbreak using temperate data from the same date in previous years for either historical context or to produce predictions for ongoing outbreaks. Fig. 6 shows both the time required for $95 \%$ of the ABS simulations to reach eradication and the degree-day thermal accumulation-based three generation time $\left(\mathrm{F}_{3}\right)$ computed using temperature values from 1950 to 2014. The values for the actual outbreak year of 2014 (filled markers) show that the degree-day calculation is exceptionally short while the ABS is almost the median value of previous years.

\section{CONCLUSION}

In the context of an area-wide programme aimed at achieving zero pest prevalence, determining the duration of eradication programmes following incursions by invasive tephritid fruit flies is as critical as it is difficult. Critical because failure to eradicate invading fruit flies will lead to increased costs incurred under follow-up programmes once population sizes increase again or are established in a different area, to say 
nothing of the costs of establishment in areas where they are not present (Siebert and Cooper 1995). In addition to these risks from control/quarantine periods that are too short, it is also important not to set overly long periods as these could be unnecessarily burdensome to producers and also lead to excessive programme costs and losses. The difficulty, however, lies in estimating the size of a possible remnant population of flies that contains so few detectable individuals (nominally adults responsive to the lure being used in the trapping array) that it is unlikely to catch any initially (Carey et al. 2017; McInnis et al. 2017).

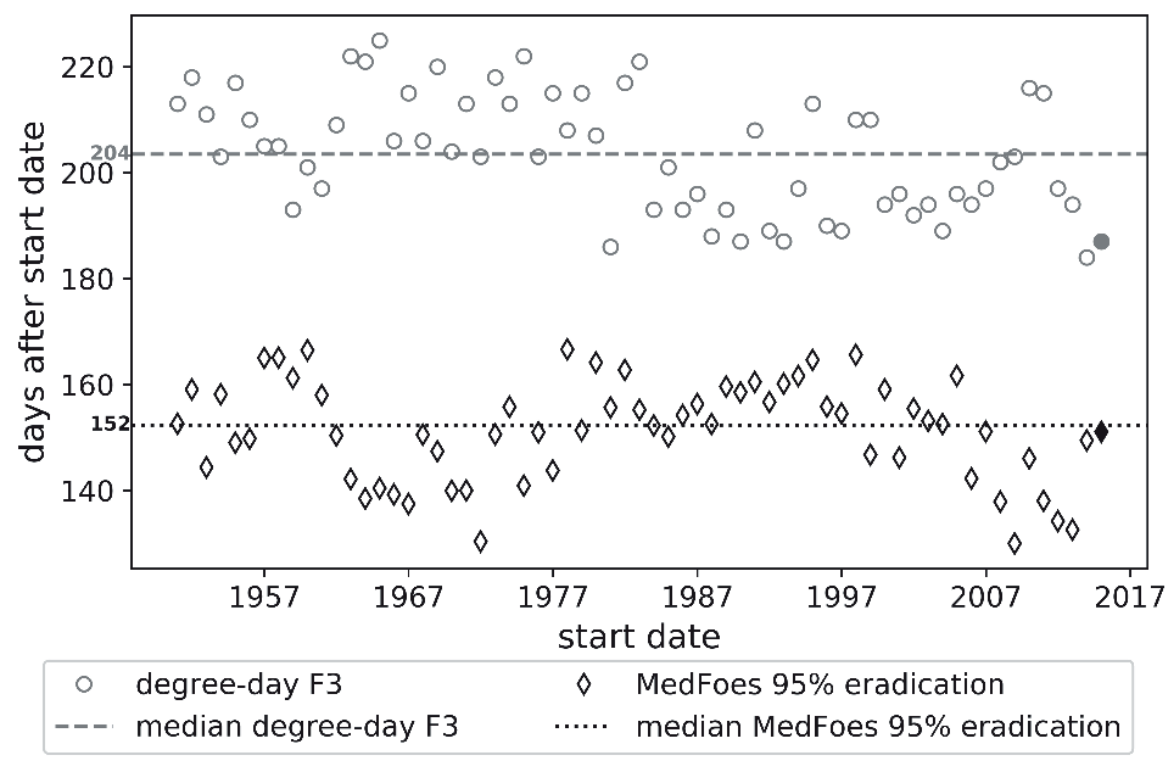

Figure 6. Times required for $95 \%$ of the ABS simulations to reach eradication in comparison to the three generation times $\left(F_{3}\right)$ based on the degree-day thermal accumulation computed for hypothetical Perris, CA outbreaks staring on 29 December for the years 1950 through 2014. The actual outbreak in 2014 is shown by filled markers. Degree-day based values are circles, and ABS based values are diamonds. Dashed lines are medians.

Though the task is difficult, the established method of using simple thermal accumulation models to guide control activity durations and ending quarantines after the equivalent of three generations of degree-day development have elapsed without another find seems to work well in practice in many places around the world, including Chile and California as described here, though there are limits in more temperate areas (Collier and Manoukis 2017).

Agent-Based Simulations, as modelled in MED-FOES, can be a useful complement for programme managers and state entities as they provide a semiindependent estimate of the duration that control and quarantine activities should continue after the detection of the last fly find. The software is relatively easy to use and with increasing computational power in multi-core desktop computers it is feasible to run a large number of simulations in a relatively short time. 
It is important to stress also the drawbacks of using ABS methods in general. These include the need for range estimates of many constituent parameters. In the case of Mediterranean fruit fly over 100 years of research on development, reproduction and mortality of this species is helpful, but some details remain poorly resolved, especially under natural conditions. A second general problem with individual-based methods is that simulating combinations of parameters to exhaustively test the range of possible outcomes can be computationally expensive. Here, again, current computer systems are powerful enough to mitigate this issue, but this problem can increase as models become more complex. A final point here is that the stochastic nature of the simulation and variable output necessitates statistical methods to handle results, and the mechanisms that drive outcomes may not be immediately clear.

One possible approach that integrates the current approach and the ABS would be to set the initial eradication programme duration following the last detection after an outbreak using the three-generation calculation on historical temperatures (already done in at least Florida and California USA, Gilbert et al. 2013), and then update with both degree-day calculations using measured hourly temperature data and with ABS simulations. The combination of both estimates will give improved insight to the outbreak dynamics, might suggest programmatic responses of effort modulation, and increase confidence in declarations of eradication.

MED-FOES instantiates a relatively simple model and therefore has several notable weaknesses. Control measures are simulated as constant average effects instead of time-varying, while eradication programmes typically incorporate discrete high-intensity efforts such as fruit removal, which will have different impacts depending on the age structure of the population. Additionally, quarantines are held for a period of time (at least one degree-day generation for C. capitata in California) after the end of suppression efforts during which intensive trapping continues. Finally, incorporating information on host plant availability, when known, would likely have significant effects. However, a major strength of the ABS approach is that all of these factors and more could be incorporated into the model in a straight-forward manner given the relevant input data.

Future developments for MED-FOES aimed at facilitating declaration of eradication after outbreaks include improved reporting incorporating analysis of historical temperature profiles. The analysis of temperatures in past years will be helpful for tracking anomalously long or short quarantine/control periods after the last detection, again increasing confidence in lengths. The flexibility and extensibility of the ABS framework also allows other applications of this model to address questions around the invasion biology of $C$. capitata.

One of the new applications of MED-FOES currently being explored is to estimate the potential growth rate of Mediterranean fruit fly populations in different areas and at different times of the year. This would yield results that could be compared to those from standard climatic suitability methods such as CLIMEX and Maxent (Webber et al. 2011). The specificity of MED-FOES to the biology of Mediterranean fruit fly could serve to refine the more general spatial and temporal analysis of patterns of detections and outbreaks when compared to the standard approaches based on environmental suitability modelling, especially when combined with incursion risk data. 


\section{ACKNOWLEDGEMENTS}

Special thanks to Ricardo Rodriguez of SAG (Chile) for information on the Santiago outbreak and permission to share our analysis. Thanks also to Kevin Hoffman of CDFA for details on the Perris, California outbreak.

\section{REFERENCES}

Almeida, S. J. de, R. P. Martins Ferreira, Á. E. Eiras, R. P. Obermayr, and M. Geier. 2010. Multiagent modeling and simulation of an Aedes aegypti mosquito population. Environmental Modelling and Software 25: 1490-1507.

Barclay, H. J., J. W. Hargrove, A. Meats, and A. Clift. 2021. Procedures for declaring pest free status, pp. 921-946. In V. Dyck, J. Hendrichs, and A. S. Robinson (eds.), Sterile Insect Technique - Principles and practice in Area-Wide Integrated Pest Management. Second Edition. CRC Press, Boca Raton, Florida, USA.

Blower, S. M., and H. Dowlatabadi. 1994. Sensitivity and uncertainty analysis of complex models of disease transmission: An HIV model, as an example. International Statistical Review 2: 229-243.

California Code of Regulations. 2017. Mediterranean fruit fly interior quarantine regulation. Title 3 Section 3406. Sacramento, California, USA.

Campbell, G. S., and J. M. Norman. 1997. An introduction to environmental biophysics, 2nd ed. Springer, New York, USA.

Carey, J. R. 1982. Demography and population dynamics of the Mediterranean fruit fly. Ecological Modeling 16: 125-150.

Carey, J. R. 1991. Establishment of the Mediterranean fruit fly in California. Science 253: 1369-1373.

Carey, J. R., N. Papadopoulos, and R. E. Plant. 2017. The 30-Year Debate on a multi-billion-dollar threat: Tephritid fruit fly establishment in California. American Entomologist 63: 100-113.

Collier, T., and N. Manoukis. 2017. Evaluation of predicted Medfly (Ceratitis capitata) quarantine length in the United States utilizing degree-day and agent-based models. F1000Research 6: 1863.

Corbett, A., and R. E. Plant. 1993. Role of movement in the response of natural enemies to agroecosystem diversification: A theoretical evaluation. Environmental Entomology 22: 519-531.

Crespo-Pérez, V., F. Rebaudo, J. F. Silvain, and O. Dangles. 2011. Modeling invasive species spread in complex landscapes: The case of potato moth in Ecuador. Landscape Ecology 26: 1447-1461.

Fletcher, B. S. 1989. Temperature-development rate relationships of the immature stages and adults of tephritid fruit flies, pp. 273-289. In A. S. Robinson, and G. Hooper (eds.), Fruit flies, their Biology, natural enemies and control. Elsevier, Amsterdam, The Netherlands.

Gilbert, A., R. Bingham, M. Nicolas, and R. Clark. 2013. Insect trapping guide, 13th edition. California Department of Food and Agriculture, Sacramento California, USA.

Gutierrez, A. P., and L. Ponti. 2011. Assessing the invasive potential of the Mediterranean fruit fly in California and Italy. Biological Invasions 13: 2661-2676.

(FAO) Food and Agriculture Organization of the United Nations. 2016a. Guidelines for pest eradication programmes. International Standard for Phytosanitary Measures (ISPM) 9. International Plant Protection Convention (IPPC). FAO, Rome, Italy.

(FAO) Food and Agriculture Organization of the United Nations. 2016b. Establishment of pest free areas for fruit flies (Tephritidae). International Standard for Phytosanitary Measures (ISPM) No. 26. International Plant Protection Convention. Rome, Italy.

Hendrichs, J., P. Kenmore. A. S. Robinson, and M. J. B. Vreysen. 2007. Area-wide integrated pest management (AW-IPM): Principles, practice and prospects, pp. 3-33. In M. J. B. Vreysen, A. S. Robinson, and J. Hendrichs (eds.), Area-wide control of insect pests: From research to field implementation. Springer, Dordrecht, The Netherlands.

Jackson, D. E., M. Holcombe, and F. L. Ratnieks. 2004. Trail geometry gives polarity to ant foraging networks. Nature 432: 907-909.

Kareiva, P. M. 1983. Local movement in herbivorous insects: Applying a passive diffusion model to markrecapture field experiments. Oecologia 57: 322-327.

Kean, J. M., P. C. Tobin, D. C. Lee, G. R. Smith, L. D. Stringer, R. F. Vargas, D. G. McCullough, D. A. Herms, J. Fletcher, D. M. Suckling, and others. 2012. Global eradication and response database. GERDA. 
Knipling, E. F. 1979. The basic principles of insect population suppression and management, Agriculture Handbook. U.S. Department of Agriculture, Washington DC, USA.

Manoukis, N. C., and K. Hoffman. 2014. An agent-based simulation of extirpation of Ceratitis capitata applied to invasions in California. Journal of Pest Science 87: 39-51.

Manoukis, N. C., B. Hall, and S. M. Geib. 2014. A computer model of insect traps in a landscape. Scientific Reports 4: 7015.

Manoukis, N. C., M. Siderhurst, and E. B. Jang. 2015. Field estimates of attraction of Ceratitis capitata to trimedlure and Bactrocera dorsalis to methyl eugenol in varying environments. Environmental Entomology 44: 695-703.

McInnis, D. O., J. Hendrichs, T. Shelly, N. Barr, K. Hoffman, R. Rodriguez, D. R. Lance, K. Bloem, D. M. Suckling, W. Enkerlin, P. Gomes, and K. H. Tan. 2017. Can polyphagous invasive tephritid pest populations escape detection for years under favorable climatic and host conditions? American Entomologist 63: 89-99.

MED-FOES. 2019. MED-FOES: MED-Fly Outbreak and Extirpation Simulation. An Agent-Based Simulation of Invasive Pest Insects.

Myers, J. H., D. Simberloff, A. M. Kuris, and J. R. Carey. 2000. Eradication revisited: Dealing with exotic species. Trends in Ecology and Evolution 15: 316-320.

Papadopoulos, N. T., R. E. Plant, and J. R. Carey. 2013. From trickle to flood: the large-scale, cryptic invasion of California by tropical fruit flies. Proceedings of the Royal Society B: Biological Sciences 280: 20131466.

Pratt, S. C., D. J. T. Sumpter, E. B. Mallon, and N. R. Franks. 2005. An agent-based model of collective nest choice by the ant Temnothorax albipennis. Animal Behaviour 70: 1023-1036.

Radchuk, V., K. Johst, J. Groeneveld, V. Grimm, and N. Schtickzelle. 2013. Behind the scenes of population viability modeling: Predicting butterfly metapopulation dynamics under climate change. Ecological Modeling 259: 62-73.

Railsback, S. F., and V. Grimm. 2012. Agent-based and individual-based modeling: A practical introduction. Princeton University Press, Princeton, New Jersey, USA.

Reicosky, D. C., L. J. Winkelman, J. M. Baker, and D. G. Baker. 1989. Accuracy of hourly air temperatures calculated from daily minima and maxima. Agricultural and Forest Meteorology 46: 193209.

Roltsch, W. J., F. G. Zalom, A. J. Strawn, J. F. Strand, and M. J. Pitcairn. 1999. Evaluation of several degree-day estimation methods in California climates. International Journal Biometeorology 42: 169_ 176.

Shelly, T. E., D.R. Lance, K.H. Tan, D.M. Suckling, K. Bloem, W. Enkerlin, K. Hoffman, N. Barr, R. Rodríguez, P.J. Gomes, and J. Hendrichs. 2017. To repeat: Can polyphagous invasive tephritid pest populations remain undetected for years under favorable climatic and host conditions? American Entomologist 63: 224-231.

Siebert, J., and T. Cooper. 1995. If medfly infestation triggered a trade ban: Embargo on California produce would cause revenue, job loss. California Agriculture 49: 7-12.

Skellam, J. G. 1951. Random dispersal in theoretical populations. Biometrika 38: 196-218.

Smith, J. W. 1998. Boll weevil eradication: Area-wide pest management. Annals of the Entomological Society of America 91: 239-247.

Suckling, D. M., J. M. Kean, L. D. Stringer, C. Cáceres-Barrios, J. Hendrichs, J. Reyes-Flores, and B. Dominiak. 2016. Eradication of tephritid fruit fly pest populations: Outcomes and prospects. Pest Management Science 72(3): 546-465.

Tassan, R. L., K. S. Hagen, A. Cheng, T. K. Palmer, G. Feliciano, and T. L. Blough. 1983. Mediterranean fruit fly life cycle estimations for the California eradication program, pp. 564-570. In R. Cavalloro (ed.), Fruit flies of economic importance. Balkema, Rotterdam, The Netherlands.

Vargas, R. I., W. A. Walsh, D. Kanehisa, E. B. Jang, and J. W. Armstrong. 1997. Demography of four Hawaiian fruit flies (Diptera: Tephritidae) reared at five constant temperatures. Annals of the Entomological Society of America 90: 162-168.

Vinatier, F., P. Tixier, P. F. Duyck, and F. Lescourret. 2011. Factors and mechanisms explaining spatial heterogeneity: A review of methods for insect populations. Methods in Ecology and Evolution 2: 1122.

Webber, B. L., C. J. Yates, D. C. Le Maitre, J. K. Scott, D. J. Kriticos, N. Ota, A. McNeill, J. J. Le Roux, and G. F. Midgley. 2011. Modelling horses for novel climate courses: Insights from projecting potential distributions of native and alien Australian acacias with correlative and mechanistic models. Diversity and Distribution 17: 978-1000. 